\title{
Asymmetric dimethylarginine in somatically healthy schizophrenia patients treated with atypical antipsychotics: a case-control study
}

Anders Jorgensen ${ }^{1,2,7^{*}}$, Ulla Knorr ${ }^{1}$, Mia Greisen Soendergaard' ${ }^{1}$, Jens Lykkesfeldt ${ }^{5}$, Anders Fink-Jensen ${ }^{1,2,5}$, Henrik Enghusen Poulsen ${ }^{3,4,5}$, Martin Balslev Jorgensen ${ }^{1,2,5}$, Niels Vidiendal Olsen ${ }^{2,6}$ and Jonatan Myrup Staals $\varnothing^{2}$

\begin{abstract}
Background: Schizophrenia is associated with increased cardiovascular morbidity and mortality. Asymmetric dimethylarginine (ADMA), an endogenous inhibitor of the nitric oxide synthase, and the L-arginine:ADMA ratio are markers of endothelial dysfunction that predict mortality and adverse outcome in a range of cardiovascular disorders. Increased ADMA levels may also lead to increased oxidative stress. We hypothesized that ADMA and the L-arginine: ADMA ratio are increased in somatically healthy schizophrenia patients treated with atypical antipsychotics (AAP), and that the ADMA and the L-arginine: ADMA ratio are positively correlated to measures of oxidative stress.

Methods: We included 40 schizophrenia patients treated with AAP, but without somatic disease or drug abuse, and 40 healthy controls. Plasma concentrations of ADMA and L-arginine were determined by high-performance liquid chromatography. Data were related to markers of systemic oxidative stress on DNA, RNA and lipids, as well as measures of medication load, duration of disease and current symptomatology.

Results: Plasma ADMA and the L-arginine:ADMA ratio did not differ between schizophrenia patients and controls. Furthermore, ADMA and the L-arginine:ADMA ratio showed no correlations with oxidative stress markers, medication load, or Positive and Negative Syndrome Scale scores.

Conclusions: Schizophrenia and treatment with AAP was not associated with increased levels of plasma ADMA or the L-arginine:ADMA ratio. Furthermore, plasma levels of ADMA were not associated with levels of systemic oxidative stress in vivo.
\end{abstract}

Keywords: Schizophrenia, Atypical antipsychotics, Asymmetric dimethylarginine, Oxidative stress

\section{Background}

Schizophrenia is associated with increased somatic morbidity and mortality, primarily due to cardiovascular disorders [1-4]. The causes for this association are thought to be poor somatic care, unhealthy lifestyle, and the side-effects of the most frequently used antipsychotic agents, known as atypical antipsychotics (AAP), which may cause weight gain and metabolic syndrome [1]. Collectively, these factors likely converge on the induction of pathophysiological pathways in the cardiovascular

\footnotetext{
* Correspondence: anders.01.joergensen@regionh.dk

'Psychiatric Centre Copenhagen, University Hospital of Copenhagen, Copenhagen, Denmark

${ }^{2}$ Department of Neuroscience and Pharmacology, Laboratory of Neuropsychiatry, University of Copenhagen, Copenhagen, Denmark Full list of author information is available at the end of the article
}

system that underlies the development of clinical cardiovascular disease in general, such as endothelial dysfunction [5]. In a recent study of schizophrenia patients treated mainly with AAP, 48 percent of the study population $(\mathrm{N}=203)$ were found to meet the criteria for endothelial dysfunction, as measured by peripheral artery tonometry [6]. Hence, endothelial dysfunction is a possible cellular phenomenon underlying the association between schizophrenia, AAP use, and cardiovascular mortality.

Nitric oxide (NO) is the main paracrine signaling molecule in the endothelial regulation of vascular smooth muscle relaxation. Three isoforms of NO-synthase catalyze the reaction arginine $+\mathrm{O}_{2}->\mathrm{NO}+$ citrulline. In conditions of arginine shortage (as well as other cofactors), the 
NO-synthases can turn to produce superoxide thus promoting oxidative stress [7]. As an endogenous inhibitor of the endothelial NO-synthase (eNOS), asymmetric dimethylarginine (ADMA) exerts its effects through a reduction in NO levels. However, increased ADMA levels have also been associated with increased oxidative stress [8-11]. ADMA administration has been shown to increase levels of reactive oxygen species in both rodent and human vasculature $[9,12]$. A shift from a NO-mediated quiescent state of the endothelium to a ROS-mediated activated and pro-inflammatory state is thought to be a key event in the transition to atherosclerosis [5].

ADMA and the L-arginine:ADMA ratio has been shown to predict mortality and adverse outcome in a range of cardiovascular disorders [5,13-16]. Both preclinical and clinical studies have shown that ADMA has a pathophysiological role in the induction of vascular dysfunction and human cardiovascular disease [11]. The L-arginine:ADMA ratio has been viewed by some authors to be a superior marker for endothelial dysfunction, as it may reflect the intracellular balance between substrate and inhibitor of NO-synthesis [17].

Plasma ADMA levels has been investigated in three previous studies of schizophrenia patients. Das et al. found that plasma ADMA were elevated in drug-naïve, first episode patients $(\mathrm{N}=16)$ compared with controls $(\mathrm{N}=12)$ [18]. Celik et al. found that plasma ADMA levels were increased in both first-episode $(\mathrm{N}=24)$ and multiple episode $(\mathrm{N}=25)$ schizophrenia patients compared with healthy controls $(\mathrm{N}=30)$. ADMA was not correlated to symptomatology, but was higher in multiple versus first-episode patients [19]. Zincir et al. found that plasma ADMA was 3 -fold higher in somatically healthy first episodeschizophrenia patients $(\mathrm{N}=49)$ compared to healthy controls $(\mathrm{N}=30)$, with a $50 \%$ reduction of plasma ADMA in the patient group after two months of treatment [20].

The purpose of the present study was to determine plasma levels of ADMA and the L-arginine:ADMA ratio in somatically healthy schizophrenia patients in treatment with AAP and healthy controls, and to compare these levels with measures of systemic oxidative stress on DNA, RNA and lipids, as well as duration of disease and symptom severity. We hypothesized that ADMA and the L-arginine: ADMA ratio are increased in schizophrenia; that the ADMA and the L-arginine:ADMA ratio are positively correlated to measures of oxidative stress in both healthy controls and schizophrenia patients; and finally that ADMA and the L-arginine:ADMA ratio are correlated to duration of disease, rather than to current symptom severity.

\section{Methods}

\section{General study outline}

Patients were recruited by referral from doctors at the Psychiatric Centre Copenhagen, which provides mental health services to the citizens of the central, northern and north-western area of Copenhagen. Inpatients and patients from the affiliated outpatient clinics were eligible for inclusion.

The inclusion criterion for patients was an ICD-10 diagnosis of schizophrenia (F20.0-F20.9) confirmed by a structured interview at referral (see below). Exclusion criteria were: 1) Somatic disease and somatic medication. A non-regular use of e.g. painkillers or asthma medication was allowed, 2) Abuse of alcohol, marihuana or other drugs of abuse, 3) Coercion of any kind, 4) Severely disorganised thinking, making it impossible to obtain an informed consent, 5) Use of dietary supplements, and 6) Pregnancy or breast-feeding. Of forty-five patients referred to the study and accepting to participate, 40 were included. In the rejected patients, the diagnosis of schizophrenia was considered uncertain after the inclusion interview $(\mathrm{N}=4)$, or the biochemical screening revealed a medical disorder $(\mathrm{N}=1)$.

Healthy controls were recruited from the blood donation corps at Rigshospitalet by personal contact, as they were scheduled for donating blood. Exclusion criteria for the healthy controls were: 1) Any psychiatric or somatic disease, 2) abuse of alcohol, marihuana or other drugs, 3) use of any medication including dietary supplements, and 4) first degree family members with psychiatric disease. A total of 175 healthy controls meeting none of the exclusion criteria were asked to participate, and of these, 40 accepted to be included.

In all participants, a Schedules in Clinical Neuropsychiatry (SCAN)-interview [21] was applied to ensure that the ICD-10 diagnostic criteria for schizophrenia were met in patients, and that no lifetime psychiatric morbidity was present in the healthy controls. The severity of psychopathology was measured (patients only) with the Positive and Negative Syndrome Scale (PANSS) [22]. The level of perceived stress was assessed with the Perceived Stress Scale 10-item (PSS) [23]. The degree of childhood adversity were estimated by the Childhood Abuse and Trauma Scale (CATS) [24]. Exercise levels (hours per week) were recorded. Exercise was defined as rigorous physical activity such as running, aerobics, sports etc.; e.g. "walking to work" or "moving a lot during the day" were not recorded as exercise. In patients, duration of disease, number of psychiatric admissions, and current psychopharmacological treatment were recorded. All patients had received a clinical examination and regular ECG's, following the standard protocol of the department, with no indication of unrecognized somatic disease.

All participants had blood drawn in the fasting state at 9 a.m. Plasma was obtained from cooled EDTA-coated tubes which were centrifuged at $4{ }^{\circ} \mathrm{C}$ and $1590 \times \mathrm{g}$ for $10 \mathrm{~min}$. Samples were stored for later analyses at $-80^{\circ} \mathrm{C}$ 
within $30 \mathrm{~min}$ from venipuncture. A spot urine sample was obtained from the first voided urine after blood sampling. Urine samples were kept on ice and transferred within hours to storage at $-20^{\circ} \mathrm{C}$ until analysis. To screen for unrecognized medical disorders and assess the cardiovascular risk profile, general biochemical tests were performed, comprising a full blood count, hepatic enzymes, total cholesterol, low-density lipoprotein (LDL) and high-density lipoprotein (HDL) cholesterol, triglycerides, c-reactive protein (CRP), thyroid stimulating hormone (TSH), sodium, potassium, creatinine, glucose, glycated hemoglobin (HbA1c), and plasma cortisol. Resting blood pressure and pulse, body weight and waist-hip ratio were recorded. The presence of metabolic syndrome was identified using criteria from the American Heart Association/National Heart, Lung, and Blood Institute [25].

\section{Plasma ADMA and L-arginine determination}

Plasma ADMA and L-arginine was determined by HighPerformance Liquid Chromatography as previously described $[26,27]$. Plasma samples underwent solid-phase extraction (Oasis MCX column, Waters) followed by high-performance liquid chromatography separation on a symmetry C18 column, $3.9 \AA \sim 15 \mathrm{~mm}, 5 \mu \mathrm{m}$ pore (Waters, WAT046980). The high-performance liquid chromatography equipment consisted of a Waters 717 plus autosampler, 2Shimadzu LC-20 AD Prominence Pumps, a Shimadzu DGU-20A5 Prominence Degasser, and a Shimadzu RF-20A Prominence Fluorescence Detector. Data collection, peak identification, and calculation of area under the curve were performed with Empower 2.0 software (Waters). Standards were purchased from Sigma-Aldrich except for monoethyl arginine (Enzo Life Sciences).

\section{Oxidative stress marker determination}

The urinary content of the oxidatively modified guanine nucleosides 8-oxo-7,8-dihydro-2'-deoxyguanosine (8-oxodG, a marker of systemic DNA damage from oxidation) and 8-oxo-7,8-dihydroguanosine (8-oxoGuo, a marker of systemic RNA damage from oxidation) were assayed using ultraperformance liquid chromatography and tandem mass spectrometry (UPLC MS/MS), as previously described [28]. The 8-oxodG/8-oxoGuo excretion is defined as the urinary concentration of the nucleoside normalized to urinary creatinine concentration [29]. Plasma malondialdehyde (MDA, a marker of lipid peroxidation) was measured, also using previously described chromatographic methods [30].

\section{Statistics}

All data are presented as means $\pm \mathrm{SD}$ or median (interquartile range). Data were compared with independent samples $t$-test, Mann-Whitney test or Chi-squared test, as appropriate. Correlation analyses were performed by Spearman or Pearson tests, as appropriate. All correlation analyses were performed in the patient and control groups separately, as well as in the full study population. 8-oxodG/8-oxoGuo deviated from normal distribution and were transformed by the natural logarithm before analyses. All statistical analyses were performed using the Statistical Package for the Social Sciences (SPSS) software version 20.0 (IBM Corporation, NY, USA). Statistical significance was defined as $\mathrm{p}<0.05$. All statistical tests were two sided.

\section{Ethics}

The study protocol complied with the Declaration of Helsinki, and was approved by the Committee on Research Ethics of the Capital Region of Denmark (H-D-2008-064) and the National Data Protection Agency of Denmark (2008-41-2052). Before inclusion, all participants were thoroughly informed on the study both in writing and orally, and all patients were offered to have a healthy relative present when the information was given. Patients with severely disorganised thinking, making it impossible to obtain an informed consent, were not invited for participation. All participants gave a written informed consent before inclusion.

\section{Results}

Baseline demographic and biochemical characteristics of the cohort are summarized in Table 1. Disease course and treatment data for the schizophrenia patients are summarized in Table 2. Note that these data have been published previously [31]. All patients received AAPs. The primary antipsychotic agents used were risperidone $(\mathrm{N}=9)$, quetiapine $(\mathrm{N}=9)$, aripiprazole $(\mathrm{N}=6)$, olanzapine $(\mathrm{N}=5)$, clozapine $(\mathrm{N}=5)$, sertindole $(\mathrm{N}=3)$, ziprasidone $(\mathrm{N}=2)$, and amisulprid $(\mathrm{N}=1)$.

We found no differences in ADMA ( $\mathrm{p}=0.80)$, L-arginine $(\mathrm{p}=0.80)$, or the L-arginine:ADMA ratio $(\mathrm{p}=0.96)$ in schizophrenia patients versus healthy controls (Figure 1). The absolute values found were: ADMA, schizophrenia: $0.37 \pm 0.08 \mu \mathrm{mol} / \mathrm{L}$, controls: $0.37 \pm 0.08 \mu \mathrm{mol} / \mathrm{L}$; L-arginine, schizophrenia: $126 \pm 22 \mu \mathrm{mol} / \mathrm{L}$, controls: $128 \pm 37 \mu \mathrm{mol} / \mathrm{L}$; L-arginine:ADMA ratio, schizophrenia: $354 \pm 79$, controls: $353 \pm 104$. The $95 \%$-confidence interval for the difference in ADMA was -0.04 to $0.03 \mu \mathrm{mol} / \mathrm{L}$ and -40 to 42 for the arginine:ADMA ratio. No significant differences were found when analyzing males and females separately (results not presented).

Next, we analyzed correlations between ADMA, the L-arginine:ADMA ratio and markers of oxidative stress (8-oxodG, 8-oxoGuo and MDA) in the full study population, as well as in the control and schizophrenia patient group separately. Results are summarized in 
Table 1 Basic data of healthy controls and schizophrenia patients

\begin{tabular}{|c|c|c|c|}
\hline & Control $(\mathrm{N}=40)$ & Schizophrenia $(\mathrm{N}=40)$ & $P$-value \\
\hline Gender (M/F) & $20 / 20$ & $20 / 20$ & 1.0 \\
\hline Age (years) & $31.4 \pm 9.8$ & $33.0 \pm 10.7$ & 0.49 \\
\hline Smoker (\%) & 21 & 55 & 0.002 \\
\hline Cigarettes per day (if smoker) & $8.1 \pm 6.4$ & $18.1 \pm 11.3$ & 0.03 \\
\hline Alcohol (drinks per week) & $5(2-10)$ & $1(0-5)$ & 0.002 \\
\hline Exercise (hours per week) & $3(1-5)$ & $0(0-2)$ & $<0.001$ \\
\hline Body Mass Index & $25.1 \pm 3.8$ & $27.2 \pm 5.7$ & 0.06 \\
\hline Waist-hip ratio & $0.80 \pm 0.07$ & $0.89 \pm 0.08$ & $<0.001$ \\
\hline Systolic blood pressure $(\mathrm{mmHg})$ & $124.6 \pm 11.6$ & $127.1 \pm 12.2$ & 0.34 \\
\hline Diastolic blood pressure $(\mathrm{mmHg})$ & $78.9 \pm 8.6$ & $78.5 \pm 7.3$ & 0.37 \\
\hline Pulse $\left(\mathrm{min}^{-1}\right)$ & $64.6 \pm 10.6$ & $81.0 \pm 14.3$ & $<0.001$ \\
\hline Metabolic Syndrome $(y / n)^{a}$ & $2 / 37$ & $11 / 27$ & $<0.001$ \\
\hline Perceived Stress Scale score & $7.7 \pm 4.2$ & $22.3 \pm 6.7$ & $<0.001$ \\
\hline Childhood Abuse and Trauma Scale score & $11(8-20)$ & $39(17-67)$ & $<0.001$ \\
\hline Total cholesterol (mmol/L) & $4.7 \pm 0.8$ & $4.9 \pm 0.9$ & 0.44 \\
\hline HDL cholesterol (mmol/L) & $1.5 \pm 0.4$ & $1.4 \pm 0.4$ & 0.40 \\
\hline LDL cholesterol (mmol/L) & $2.8 \pm 0.6$ & $2.8 \pm 0.8$ & 0.75 \\
\hline Triglycerides (mmol/L) & $0.90(0.70-1.15)$ & $1.25(0.76-2.00)$ & 0.02 \\
\hline Glucose (mmol/L) & $5.2 \pm 0.4$ & $5.1 \pm 0.6$ & 0.48 \\
\hline $\mathrm{HbA1c}(\mathrm{mmol} / \mathrm{L})$ & $5.2 \pm 0.3$ & $5.4 \pm 0.4$ & 0.01 \\
\hline Plasma Creatinine $(\mu \mathrm{mol} / \mathrm{L})$ & $73 \pm 16$ & $69 \pm 14$ & 0.20 \\
\hline CRP (mg/L) & $1(1-2)$ & $2(1-4)$ & 0.002 \\
\hline
\end{tabular}

Numbers are means \pm standard deviation or medians (interquartile range). Data are analyzed with t-tests, Mann Whitney tests or Chi-squared tests, as appropriate. ${ }^{a}$ As defined in [25] (because of a few participants with missing values, the sum does not reach 40 in each group). Abbreviations: $H D L$ High-density lipoprotein; $L D L$ Low-density lipoprotein, HbA1c Glycated hemoglobin. CRP: C-reactive protein.

Table 3. We found no significant correlations between the ADMA and oxidative stress markers (Table 3).

There were no significant differences in ADMA or the L-arginine:ADMA ratio in metabolic syndrome versus non-metabolic syndrome individuals, neither in the the schizophrenia patients, the healthy controls, or when analyzing the population as a whole. Furthermore, we found no significant correlations between levels of exercise (hours per week) and ADMA or the L-arginine/ ADMA ratio in patients or controls, respectively (results not presented).

Because psychological stress and childhood adversity has been linked to cardiovascular disease [32,33], we further analyzed correlations between ADMA, the L-arginine: ADMA ratio, PSS and CATS scores in the full study population as well as in the control and schizophrenia patient group separately. We found no significant associations between the ADMA markers and measures of subjective current psychological stress or adverse childhood experiences (results not presented).

Finally, in patients only, we analyzed the correlations between ADMA, the L-arginine:ADMA ratio, and measures of current psychopathology, duration of disease, number of lifetime admissions, AAP doses, and duration of AAP treatment (Table 4). We found a trend towards a positive correlation between the L-arginine:ADMA ratio and duration of disease $(\mathrm{p}=0.066)$ as well as number of lifetime admissions $(\mathrm{p}=0.052)$. Otherwise, there were no significant associations between ADMA or the L-arginine: ADMA ratio and measures of current psychopathology and disease course. Specifically, none of the markers showed associations to present and past AAP use.

\section{Discussion}

This study suggests that schizophrenia patients treated with AAPs do not have alterations in ADMA and the L-arginine:ADMA ratio. The lack of association could perhaps be explained by the fact that the patients investigated in the present study were on average relatively young, and participants were excluded if they had any manifest somatic illness. Many of the schizophrenia patients did, however, exhibit a mild cardiovascular risk profile, with more patients than controls having dyslipidemia, central obesity, fulfilling criteria for the metabolic syndrome, etc. The prevalence of the metabolic syndrome was even slighty higher than the one found in a 
Table 2 Clinical characteristics of the schizophrenia patients. Numbers are means \pm standard deviation or medians (interquartile range)

\begin{tabular}{|c|c|}
\hline Diagnosis, ICD-10 & \\
\hline Paranoid schizophrenia & $55 \%$ \\
\hline Undifferentiated schizophrenia & $28 \%$ \\
\hline Hebephrenic schizophrenia & $12 \%$ \\
\hline Unspecified schizophrenia & $5 \%$ \\
\hline \multicolumn{2}{|l|}{ Disease course } \\
\hline Age of onset (years) & $23.0 \pm 5.3$ \\
\hline Duration of illness (months) & $76(36-189)$ \\
\hline Number of admissions & $3(1-6)$ \\
\hline In-patient at inclusion & $60 \%$ \\
\hline \multicolumn{2}{|l|}{ Medication $^{\mathrm{a}}$} \\
\hline Duration of current antipsychotic treatment (months) & $20(3-68)$ \\
\hline \multicolumn{2}{|l|}{ Number of antipsychotics used } \\
\hline One & $77 \%$ \\
\hline Two or three & $23 \%$ \\
\hline Antipsychotics, Defined Daily Dose ${ }^{b}$ & $1.5(0.7-2.3)$ \\
\hline Antidepressant use & $43 \%$ \\
\hline If antidepressant use, Defined Daily Dose & $1.4(1.0-2.0)$ \\
\hline Benzodiazepine use & $28 \%$ \\
\hline If benzodiazepine use, Defined Daily Dose & $0.6(0.3-1.2)$ \\
\hline \multicolumn{2}{|l|}{ Psychopathology } \\
\hline PANSS positive score & $22.2 \pm 4.3$ \\
\hline PANSS negative score & $22.3 \pm 5.6$ \\
\hline PANSS general score & $44.6 \pm 8.5$ \\
\hline PANSS total score & $89.1 \pm 16.5$ \\
\hline
\end{tabular}

recent large meta-analyses [34]. Therefore, it is surprising that we were unable to identify any differences in ADMA, which has previously been associated with a cardiovascular risk profile [5]. Due to the complete absence of borderline significant trends, it is not likely that the findings are due to the medium size of the study, i.e. due to a type II error. The 95\% confidence limits for the difference in plasma ADMA were -0.04$0.03 \mu \mathrm{mol} / \mathrm{L}$, implying that any potential difference that we may have overlooked is likely to be within this range, which must be considered to be clinically unimportant.

As an endogenous inhibitor of the nitric oxide synthases, ADMA is a mediator of endothelial dysfunction with modest correlation to peripheral tonometry [26], and low correlation to flow mediated dilation [35]) which are measures of endothelial dysfunction. Thus, it is possible that some degree of endothelial dysfunction have been overlooked in the present population. However,

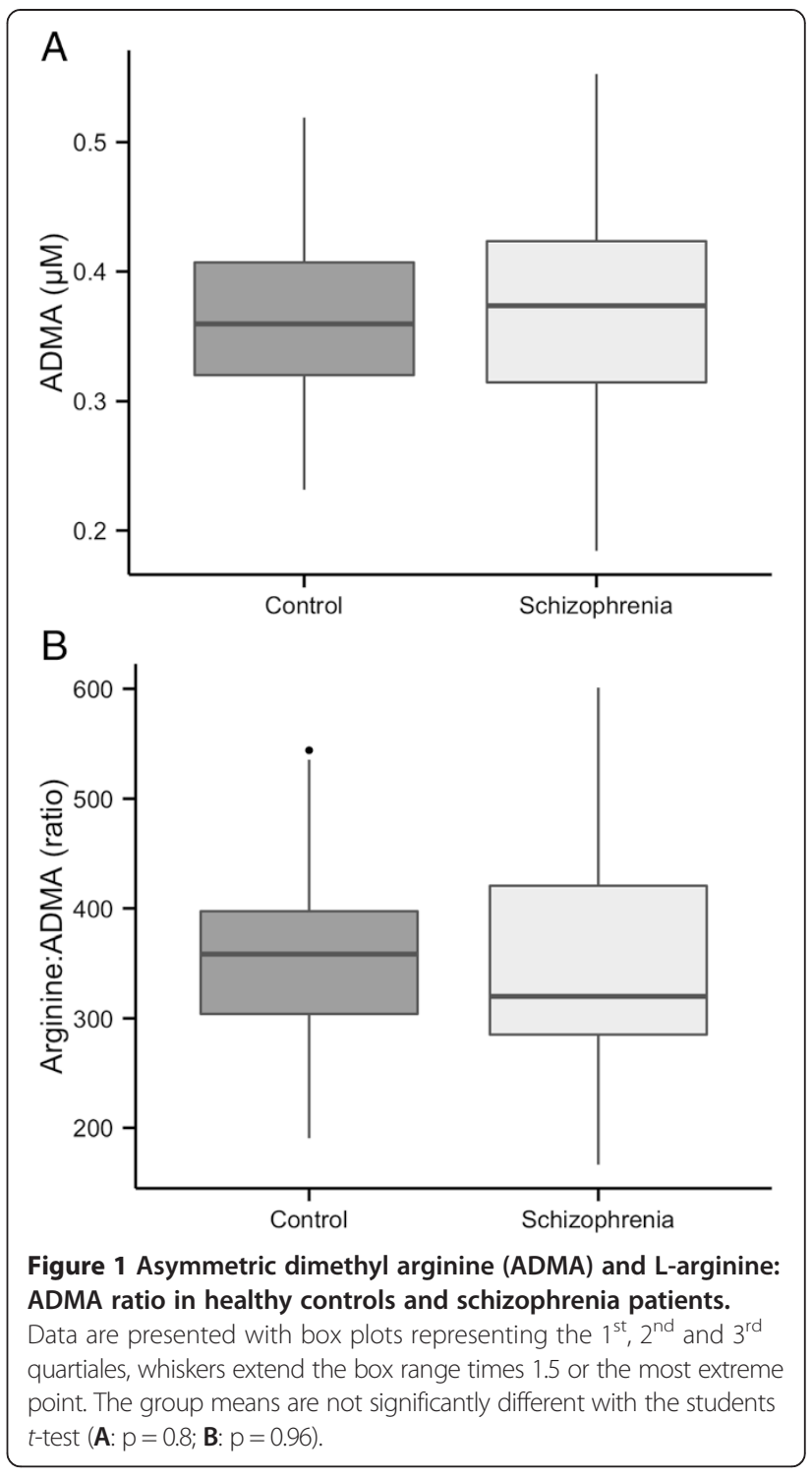

ADMA is near the end in a chain of molecular events leading to endothelial dysfunction, and we therefore speculate that endothelial dysfunction occurs in association to more manifest cardiovascular risk or disease states than the ones present in our patient population.

The finding is in contrast with the findings of Das et al., Celik et al. and Zincir et al. [18-20], who found that ADMA was increased in schizophrenia patients. These studies also used HPLC to measure ADMA. In the study by Celik et al., first episode and multiple episode patients were either drug-naïve or had not received antipsychotics at least 3 months prior to blood sampling [19]. Das et al. found a reduction in ADMA after the initiation of neuroleptic treatment in a subsample of their drug-naïve patients [18]. Zincir et al. found a 50\% reduction of plasma ADMA in first episodeschizophrenia patients after two months of treatment [20]. 
Table 3 Correlations between ADMA, L-arginine:ADMA ratios, and markers of oxidatively generated DNA (8-oxodG), RNA (8-oxoGuo) and lipid (MDA) damage in all participants, healthy controls, and schizophrenia patients

\begin{tabular}{llll}
\hline & 8-oxodG & 8-oxoGuo & MDA \\
\hline All participants & $-0.08(-0.30$ to 0.14$)$ & $0.01(-0.21$ to 0.22$)$ & $-0.109(-0.33$ to 0.12$)$ \\
ADMA & $0.07(-0.16$ to 0.28$)$ & $-0.02(-0.24$ to 0.20$)$ & $0.07(-0.17$ to 0.29$)$ \\
$\begin{array}{l}\text { L-arginine:ADMA ratio } \\
\text { Healthy controls }\end{array}$ & $-0.11(-0.41$ to 0.21$)$ & $-0.11(0.41$ to 0.20$)$ & $-0.19(-0.49$ to 0.14$)$ \\
ADMA & $-0.07(-0.38$ to 0.24$)$ & $-0.08(-0.38$ to 0.24$)$ & $0.12(-0.22$ to 0.43$)$ \\
L-arginine:ADMA ratio & & $0.08(-0.24$ to 0.38$)$ & $-0.06(-0.37$ to 0.26$)$ \\
Schizophrenia patients & $-0.09(-0.39$ to 0.23$)$ & $0.02(-0.29$ to 0.33$)$ & $0.04(-0.28$ to 0.35$)$ \\
ADMA & $0.16(-0.16$ to 0.45$)$ &
\end{tabular}

Data are presented as Pearson correlation coefficients and corresponding 95\% confidence intervals. Abbreviations: ADMA Asymmetric dimethyl arginine 8-oxodG 8-oxo-7,8-dihydro-2'-deoxyguanosine, 8-oxoGuo 8-oxo-7,8-dihydroguanosine MDA Malondialdehyde.

Hence, it could be speculated that treatment with AAP's offers a short-term protection against endothelial dysfunction, perhaps by reducing acute agitation and autonomic activation. However, we did not find any significant correlations with the objective or subjective clinical severity of disease, contradicting such a hypothesis. Likewise, we found a trend towards higher levels of the L-arginine: ADMA ratio in patients with longer disease duration and a higher number of psychiatric admissions, i.e. in the opposite direction of what would be expected if the cumulative load of disease negatively influenced endothelial function. Finally, the AAP dose or duration of AAP use were not correlated to the ADMA markers, indicating that the degree of exposure to AAPs does not influence ADMA levels. Of note, in the studies by Celic et al. and Zincir et al., the reported levels af ADMA were approximately x 10 higher than the ones found in our study, suggesting that the detected substances are not identical. The levels found in our study are in line with the reference ranges suggested by others [36-38].

In the same cohort, we have previously established substantial differences in markers of systemic oxidative stress between patients and controls [31]. In this study, we found no significant correlations between ADMA or arginine:ADMA and three markers of systemic oxidatively generated damage to DNA (8-oxodG), RNA (8-oxoGuo) and lipids, neither in the individual groups nor in the cohort as a whole. To our knowledge, this is the first human study to assess the correlation between ADMA and oxidative stress markers in vivo. It is not known which tissues contribute the most to urinary and plasma markers of oxidative stress (8-oxodG/8-oxoGuo and MDA, respectively), and it may very well be that a subcellular interaction between ADMA and ROS in the endothelium does not manifest itself on the systemic level.

There are some limitations that should be mentioned. We did not include a population of patients who were not treated with AAPs, and this limits our ability to make conclusions on the relation between ADMA and AAP treatment. Further, the study did not include an alternative measure of endothelial dysfunction, such as peripheral artery tonometry. Finally, the study was cross-sectional, limiting the extent to which causal inferences can be made. Hence, we are unable to control for differences in i.e. smoking and diet, and although the differences in these factors between patients and controls would be expected to promote endothelial dysfunction and increased ADMA in patients, we cannot exclude the possibility that they could have influenced the results.

Table 4 Correlations between measures of current psychopathology, disease course and AAP use in schizophrenia patients

\begin{tabular}{lll}
\hline & ADMA & L-arginine:ADMA ratio \\
\hline PANSS score & $0.16(-0.16$ to 0.45$) p=0.31$ & $0.13(-0.19$ to 0.42$) p=0.44$ \\
Duration of disease & $-0.04(-0.34$ to 0.28$) p=0.82$ & $0.29(-0.02$ to 0.55$) p=0.07$ \\
Lifetime admissions & $-0.06(-0.37$ to 0.25$) p=0.70$ & $0.32(0.00$ to 0.57$) p=0.05$ \\
Defined Daily Dose of AAP & $0.17(-0.15$ to 0.46$) p=0.30)$ & $0.004(-0.31$ to 0.32$) p=0.98$ \\
Duration of AAP treatment & $0.14(-0.18$ to 0.43$) p=0.40$ & $-0.16(-0.45$ to 0.16$) p=0.32$ \\
\hline
\end{tabular}

Data are presented as Spearman correlation coefficients $(\rho)$ with 95-\% confidence limits (Fisher's z-transformation with normal approximation) and corresponding p-values. Abbreviations: ADMA Asymmetric dimethyl arginine, PANSS Positive and Negative Syndrome Scale, AAP Atypical antipsychotics. 


\section{Conclusions}

In conclusion, plasma ADMA and the L-arginine:ADMA ratio are not increased outside clinically relevant ranges by schizophrenia and treatment with AAP. Further, we found no evidence that ADMA or the L-arginine:ADMA ratio is associated to markers of systemic DNA, RNA and lipid damage from oxidation. The study contradicts previous studies on plasma ADMA in schizophrenia patients, and to our knowledge is the first human study to assess the relation between ADMA and markers of oxidative stress in vivo.

\section{Competing interests}

UK has been a speaker for Servier and a consultant for AstraZeneca. None of the remaining authors report any financial or other conflicts of interests.

\section{Authors' contributions}

AJ, UK, and MGS recruited the participants and performed the clinical assessments. JL, HEPO, NVO, and JMS performed the laboratory analyses. AJ, AFJ, NVO, HEPO, MBJ and JMS conceived and designed the study. AJ and JMS performed the statistical analyses and wrote the first draft of the paper All authors contributed to and approved the final version of the paper.

\section{Acknowledgements}

The study was funded by the Psychiatric Centre Copenhagen and grants from the Mental Health Services of the Capital Region of Denmark, The Ivan Nielsen Foundation, The Marie \& Børge Krogh Foundation, M.D. Gerhard Linds grant, The Psyciatric Research Foundation of 1967 and The Eli \& Egon Larsen Foundation.

We wish to thank all the participants. We further thank Drs Jeanett Bauer and Lars Søndergaard for the referral of patients, and laboratory technician Allan Hansen for technical assistance.

\section{Author details}

${ }^{1}$ Psychiatric Centre Copenhagen, University Hospital of Copenhagen, Copenhagen, Denmark. ²Department of Neuroscience and Pharmacology, Laboratory of Neuropsychiatry, University of Copenhagen, Copenhagen, Denmark. ${ }^{3}$ Laboratory of Clinical Pharmacology Q7642, Rigshospitalet, Copenhagen, Denmark. ${ }^{4}$ Department of Clinical Pharmacology, Bispebjerg Hospital, Copenhagen, Denmark. ${ }^{5}$ Faculty of Health and Medical Sciences, University of Copenhagen, Copenhagen, Denmark. ${ }^{6}$ Department of Neuroanaesthesia, The Neuroscience Centre, University Hospital of Copenhagen, Rigshospitalet, Copenhagen, Denmark. ${ }^{7}$ Psychiatric Centre Copenhagen, University Hospital of Copenhagen, Rigshospitalet, Blegdamsvej 9. DK-2100 Copenhagen, Denmark.

Received: 23 October 2014 Accepted: 23 March 2015

Published online: 03 April 2015

\section{References}

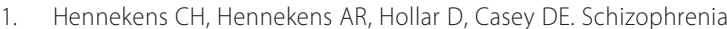
and increased risks of cardiovascular disease. Am Heart J. 2005:150(6):1115-21.

2. Hoang U, Stewart R, Goldacre MJ. Mortality after hospital discharge for people with schizophrenia or bipolar disorder: retrospective study of linked English hospital episode statistics, 1999-2006. BMJ. 2011:343:d5422.

3. Laursen TM, Munk-Olsen T, Nordentoft M, Mortensen PB. Increased mortality among patients admitted with major psychiatric disorders: a register-based study comparing mortality in unipolar depressive disorder, bipolar affective disorder, schizoaffective disorder, and schizophrenia. J Clin Psychiatry. 2007:68(6):899-907.

4. Crump C, Winkleby MA, Sundquist K, Sundquist J. Comorbidities and Mortality in Persons With Schizophrenia: A Swedish National Cohort Study. Am J Psychiatry. 2013;170(3):324-33. doi:10.1176/appi.ajp.2012.12050599.

5. Deanfield JE, Halcox JP, Rabelink TJ. Endothelial function and dysfunction: testing and clinical relevance. Circulation. 2007;115(10):1285-95.
6. Burghardt K, Grove T, Ellingrod V. Endothelial nitric oxide synthetase genetic variants, metabolic syndrome and endothelial function in schizophrenia. J Psychopharmacol. 2014;28(4):349-56. doi:10.1177/0269881113516200. Epub 2013 Dec 17

7. Forstermann U, Sessa WC. Nitric oxide synthases: regulation and function Eur Heart J. 2012;33(7):829-37. 837a-837d.

8. Sydow K, Munzel T. ADMA and oxidative stress. Atheroscler Suppl. 2003:4(4):41-51.

9. Suda O, Tsutsui M, Morishita T, Tasaki H, Ueno S, Nakata S, et al. Asymmetric dimethylarginine produces vascular lesions in endothelial nitric oxide synthase-deficient mice: involvement of renin-angiotensin system and oxidative stress. Arterioscler Thromb Vasc Biol. 2004;24(9):1682-8.

10. Korandji C, Zeller M, Guilland JC, Collin B, Lauzier B, Sicard P, et al. Time course of asymmetric dimethylarginine (ADMA) and oxidative stress in fructose-hypertensive rats: a model related to metabolic syndrome. Atherosclerosis. 2011;214(2):310-5

11. Boger $\mathrm{RH}$. Asymmetric dimethylarginine (ADMA): a novel risk marker in cardiovascular medicine and beyond. Ann Med. 2006;38(2):126-36.

12. Mohan S, Fung HL. Mechanism of cellular oxidation stress induced by asymmetric dimethylarginine. Int J Mol Sci. 2012;13(6):7521-31.

13. Pikula A, Boger RH, Beiser AS, Maas R, DeCarli C, Schwedhelm E, et al. Association of plasma ADMA levels with MRI markers of vascular brain injury: Framingham offspring study. Stroke. 2009;40(9):2959-64.

14. Anderssohn M, Rosenberg M, Schwedhelm E, Zugck C, Lutz M, Luneburg N, et al. The L-Arginine-asymmetric dimethylarginine ratio is an independent predictor of mortality in dilated cardiomyopathy. J Card Fail. 2012:18(12):904-11.

15. Duckelmann C, Mittermayer F, Haider DG, Altenberger J, Eichinger J, Wolzt M. Asymmetric dimethylarginine enhances cardiovascular risk prediction in patients with chronic heart failure. Arterioscler Thromb Vasc Biol. 2007;27(9):2037-42

16. Loland KH, Bleie O, Borgeraas H, Strand E, Ueland PM, Svardal A, et al. The association between progression of atherosclerosis and the methylated amino acids asymmetric dimethylarginine and trimethyllysine. PLoS One. 2013;8(5):e64774

17. Seljeflot I, Nilsson BB, Westheim AS, Bratseth $V$, Arnesen $H$. The L-arginine-asymmetric dimethylarginine ratio is strongly related to the severity of chronic heart failure. No effects of exercise training. J Cardiac Failure. 2011;17(2):135-42.

18. Das I, Khan NS, Puri BK, Hirsch SR. Elevated endogenous nitric oxide synthase inhibitor in schizophrenic plasma may reflect abnormalities in brain nitric oxide production. Neurosci Lett. 1996:215(3):209-11.

19. Celik C, Cayci T, Ozdemir B, Akgul EO, Zincir S, Balikci A, et al. Plasma asymmetric dimethylarginine (ADMA) concentrations in patients with first and multiple episode schizophrenia. Psychiatry Res. 2011;190(2-3):177-80.

20. Zincir S, Zincir SB, Doruk A, Erdem M, Celik C, Ak M, et al. Asymmetric dimethylarginine (ADMA) and treatment response relationship in male patients with first-episode schizophrenia: A controlled study. Psychiatry Res 2014:220(1-2):76-80. doi:10.1016/j.psychres.2014.07.013. Epub 2014 Jul 23.

21. Wing JK, Babor T, Brugha T, Burke J, Cooper JE, Giel R, et al. SCAN Schedules for Clinical Assessment in Neuropsychiatry. Arch Gen Psychiatry. 1990;47(6):589-93.

22. Kay SR, Fiszbein A, Opler LA. The positive and negative syndrome scale (PANSS) for schizophrenia. Schizophr Bull. 1987;13(2):261-76.

23. Cohen S, Williamson G. Perceived stress in a probability sample if the United States. In: SSpacapan \& SOskamp, editor. The social psychology of health: Claremont Symposium on applied social psychology. Newbury Park, CA: Sage; 1988.

24. Sanders B, Becker-Lausen E. The measurement of psychological maltreatment: early data on the Child Abuse and Trauma Scale. Child Abuse Negl. 1995;19(3):315-23

25. Grundy SM, Cleeman JI, Daniels SR, Donato KA, Eckel RH, Franklin BA, et al. Diagnosis and management of the metabolic syndrome: an American Heart Association/National Heart, Lung, and Blood Institute scientific statement. Curr Opin Cardiol. 2006;21(1):1-6.

26. Bergstrom A, Staalso JM, Romner B, Olsen NV Impaired endothelial function after aneurysmal subarachnoid haemorrhage correlates with arginine: asymmetric dimethylarginine ratio. $\mathrm{Br}$ J Anaesth. 2014;112(2):311-8.

27. Staalso JM, Bergstrom A, Edsen T, Weikop P, Romner B, Olsen NV. Low plasma arginine:asymmetric dimethyl arginine ratios predict mortality after intracranial aneurysm rupture. Stroke. 2013;44(5):1273-81. 
28. Henriksen T, Hillestrom PR, Poulsen HE, Weimann A. Automated method for the direct analysis of 8-oxo-guanosine and 8-oxo-2'-deoxyguanosine in human urine using ultraperformance liquid chromatography and tandem mass spectrometry. Free Radic Biol Med. 2009;47(5):629-35.

29. Evans MD, Olinski R, Loft S, Cooke MS. Toward consensus in the analysis of urinary 8-oxo-7,8-dihydro-2'-deoxyguanosine as a noninvasive biomarker of oxidative stress. FASEB J. 2010;24(4):1249-60.

30. Lykkesfeldt J. Malondialdehyde as biomarker of oxidative damage to lipids caused by smoking. Clin Chim Acta. 2007;380(1-2):50-8.

31. Jorgensen A, Broedbaek K, Fink-Jensen A, Knorr U, Greisen Soendergaard M, Henriksen T, et al. Increased systemic oxidatively generated DNA and RNA damage in schizophrenia. Psychiatry Res. 2013;209(3):417-23.

32. Brotman DJ, Golden SH, Wittstein IS. The cardiovascular toll of stress. Lancet 2007;370(9592):1089-100

33. Batten SV, Aslan M, Maciejewski PK, Mazure CM. Childhood maltreatment as a risk factor for adult cardiovascular disease and depression. J Clin Psychiatry. 2004;65(2):249-54.

34. Mitchell AJ, Vancampfort D, Sweers K, van Winkel R, Yu W, De Hert M. Prevalence of metabolic syndrome and metabolic abnormalities in schizophrenia and related disorders-a systematic review and meta-analysis. Schizophr Bull. 2013;39(2):306-18.

35. Juonala M, Viikari JS, Alfthan G, Marniemi J, Kahonen M, Taittonen L, et al. Brachial artery flow-mediated dilation and asymmetrical dimethylarginine in the cardiovascular risk in young Finns study. Circulation. 2007;116(12):1367-73.

36. Atzler D, Schwedhelm E, Nauck M, Ittermann T, Boger RH, Friedrich N. Serum reference intervals of homoarginine, ADMA, and SDMA in the Study of Health in Pomerania. Clin Chem Lab Med. 2014;52(12):1835-42. doi:10.1515/cclm-2014-0314.

37. El-Khoury JM, Bunch DR, Reineks E, Jackson R, Steinle R, Wang S. A simple and fast liquid chromatography-tandem mass spectrometry method for measurement of underivatized L-arginine, symmetric dimethylarginine, and asymmetric dimethylarginine and establishment of the reference ranges. Anal Bioanal Chem. 2012:402(2):771-9.

38. Luneburg N, Xanthakis V, Schwedhelm E, Sullivan LM, Maas R, Anderssohn M, et al. Reference intervals for plasma $\mathrm{L}$-arginine and the $\mathrm{L}$-arginine:asymmetric dimethylarginine ratio in the Framingham Offspring Cohort. J Nutr. 2011;141(12):2186-90

\section{Submit your next manuscript to BioMed Central and take full advantage of:}

- Convenient online submission

- Thorough peer review

- No space constraints or color figure charges

- Immediate publication on acceptance

- Inclusion in PubMed, CAS, Scopus and Google Scholar

- Research which is freely available for redistribution 